PROCEEDINGS OF THE

AMERICAN MATHEMATICAL SOCIETY

Volume 133, Number 12, Pages 3609-3611

S 0002-9939(05)07930-X

Article electronically published on June 28, 2005

\title{
A REMARK ON THE HOMOMORPHISM ON $C(X)$
}

\author{
Z. ERCAN AND S. ONAL
}

(Communicated by Joseph A. Ball)

\begin{abstract}
Let $X$ be a real compact space. Without using the axiom of choice we present a simple and direct proof that a non-zero homomorphism on $C(X)$ is determined by a point.
\end{abstract}

As usual $C(X)$ stands for the algebra of real valued continuous functions on a topological space $X$ under the pointwise operations, that is, for each $f, g, h \in C(X)$ and $\alpha \in \mathbb{R}$,

$$
(f+g)(x)=f(x)+g(x), \quad(f g)(x)=f(x) g(x) \quad \text { and } \quad(\alpha f)(x)=\alpha f(x) .
$$

A map $\varphi: C(X) \longrightarrow \mathbb{R}$ is called an (algebra) homomorphism if

$$
\varphi(f g+\alpha h)=\varphi(f) \varphi(g)+\alpha \varphi(h)
$$

for each $f, g, h \in C(X)$ and $\alpha \in \mathbb{R}$. In [4] it is shown that a map $\varphi: C(X) \longrightarrow \mathbb{R}$ is a homomorphism if and only if it is a ring homomorphism, that is,

$$
\varphi(f g+h)=\varphi(f) \varphi(g)+\varphi(h)
$$

for each $f, g, h \in C(X)$. For each $r \in \mathbb{R}, \mathbf{r}: X \longrightarrow \mathbb{R}$ is defined by $\mathbf{r}(x)=r$. The characteristic function of $A \subset X$ is denoted by $\chi_{A}$.

A Hausdorff topological space $X$ is called a real compact space if it is homeomorphic to a closed subspace of the product space $\prod_{i \in I} \mathbb{R}$ for some index set $I$. For each $j \in I, P_{j}: \prod_{i \in I} \mathbb{R} \longrightarrow \mathbb{R}$ is defined by $P_{j}\left(\left(x_{i}\right)\right)=x_{j}$ and $e_{j}=\left(x_{i}\right)$ with $x_{j}=1$ and $x_{i}=0$ if $i \neq j$.

The following theorem is well known.

Theorem 1. Let $X$ be a real compact space and let $\varphi: C(X) \longrightarrow \mathbb{R}$ be a nonzero map. Then $\varphi$ is a homomorphism if and only if there exists a unique $c \in X$ such that $\varphi(f)=f(c)$ for each $f \in C(X)$.

The known proofs of the above theorem depend on Stone-Cech compactification or (equivalently) on the maximal ideal theory (see [2, p. 241, or [3], p. 142). In [1] a simple and direct proof of the above theorem is given for $X=\mathbb{R}$ by only using properties of continuous functions. More precisely, Aron and Fricke proved that

$$
\varphi(f)=f(\varphi(i))
$$

where $i: \mathbb{R} \longrightarrow \mathbb{R}$ is the identity map, that is, $i(x)=x$. By following the technique of [1] we can reprove the above theorem simply and directly without using any

Received by the editors July 12, 2004 and, in revised form, July 30, 2004.

2000 Mathematics Subject Classification. Primary 46J10, 46 E25.

Key words and phrases. Real compact, algebra homomorphism.

(C)2005 American Mathematical Society Reverts to public domain 28 years from publication 
compactification or ideal theory. We believe that this proof may be of interest because of its simplicity and accessibility.

Proof of Theorem 1. First we prove the theorem for $X=\prod_{i \in I} \mathbb{R}$. Let

$$
c=\left(c_{i}\right)=\left(\varphi\left(P_{i}\right)\right) .
$$

We show that for each $f \in C(X)$

$$
\varphi(f)=f(c) .
$$

For each $f \in C(X)$ let

$$
k_{f}: X \longrightarrow \mathbb{R} \text { be defined by } k_{f}=f-f(c) \mathbf{1} .
$$

Then $\varphi\left(k_{f}\right)=\varphi(f)-f(c)$ and

$$
\varphi\left(k_{f}\right)=0 \Longleftrightarrow \varphi(f)=f(c) .
$$

Hence it is enough to show that

$$
f(c)=0 \Longrightarrow \varphi(f)=0 .
$$

We claim that if $\left.f\right|_{U} \equiv 0$ for some open set $U$ which contains $c$, then $\varphi(f)=0$. There exists a family $\left(U_{i}\right)_{i \in I}$ of open subsets of $\mathbb{R}$ such that

$$
c \in \prod_{i} U_{i}=V \subset U \text { and } F=\left\{i \in I: U_{i} \neq \mathbb{R}\right\} \quad \text { is finite. }
$$

Let

$$
h=\sum_{i \in F}\left(P_{i}-\mathbf{c}_{\mathbf{i}}\right)^{2} .
$$

It is clear that $h(x) \neq 0$ whenever $x \notin V$. Define

$$
g: X \longrightarrow \mathbb{R}, \quad g(x)=\frac{f(x)}{h(x)} \chi_{X \backslash V} .
$$

Then $g \in C(X)$ and

$$
f=g h .
$$

This implies that

$$
\varphi(f)=\varphi(g) \varphi(h)=\varphi(g)\left(\sum_{i \in F}\left(\varphi\left(P_{i}\right)-c_{i}\right)^{2}\right)=0 .
$$

Now suppose that there exists $f \in C(X)$ with $\varphi(f)=2$ and $f(c)=0$. Let

$$
g: \mathbb{R} \longrightarrow \mathbb{R} \text { be defined by } g(x)=-\chi_{(-\infty,-1]}(x)+x \chi_{(-1,1]}(x)+\chi_{(1, \infty)}(x) \text {. }
$$

Then $g \in C(\mathbb{R})$. Let $U=f^{-1}(-1,1)$. Then $c \in U$ and

$$
f-g \circ f=0 \text { on } U \text {. }
$$

From the above observation $2=\varphi(f)=\varphi(g \circ f)$. This implies that

$$
1=\varphi\left((\mathbf{2}-g \circ f)^{-1}(\mathbf{2}-g \circ f)\right)=\varphi\left((\mathbf{2}-g \circ f)^{-1}\right)(\varphi(\mathbf{2})-\varphi(g \circ f))=0,
$$

which is a contradiction. Clearly this shows that $\varphi(f)=0$ whenever $f(c)=0$. Now suppose that $X$ is homeomorphic to a closed subset $Y=\prod_{i \in I} \mathbb{R}$ for some set $I$. Let

$$
\psi: C(Y) \longrightarrow C(X) \text { be defined by } \psi(f)=\left.f\right|_{X},
$$

where $\left.f\right|_{X}$ denotes the restriction of $f$ to $X$. Then there exists $c \in Y$ such that

$$
\varphi \circ \psi(f)=f(c) \text { for each } f \in C(Y) .
$$


Suppose that $c \notin X$. As $Y$ is a completely regular Hausdorff space, there exists $f \in C(Y)$ such that

$$
f(c)=1 \text { and }\left.f\right|_{X} \equiv 0 .
$$

This implies that

$$
1=f(c)=\varphi \circ \psi(f)=\varphi\left(\left.f\right|_{X}\right)=0,
$$

which is a contradiction and completes the proof.

The proofs of the following corollaries immediately follow from the theorem.

Corollary 2. Let $F(\mathbb{N})$ be the algebra of real valued functions on $\mathbb{N}$ with respect to the pointwise operations. For each non-zero homomorphism $\varphi: F(\mathbb{N}) \longrightarrow \mathbb{R}$ there exists a unique $n \in \mathbb{N}$ such that $\varphi(f)=f(n)$ for each $f \in F(\mathbb{N})$.

Corollary 3. Let $X$ be a real compact Hausdorff space and let $Y$ be a completely regular Hausdorff space. Let $\varphi: C(X) \longrightarrow C(Y)$ be a non-zero homomorphism. Then there exists a continuous function $\sigma: Y \longrightarrow X$ such that

$$
\varphi(f)=f \circ \sigma .
$$

The following corollary is known as the Banach-Stone theorem.

Corollary 4. Let $X$ be a real compact space and let $Y$ be a completely regular Hausdorff space. Then $C(X)$ and $C(Y)$ are ring isomorphic if and only if $X$ and $Y$ are homeomorphic.

\section{REFERENCES}

[1] R.M. Aron and G. H. Fricke, Homomorphism on $C(\mathbb{R})$, Amer. Math. Monthly 93 (1986), no.7, 555. MR0856297 (87i:46109)

[2] R. Engelking, General Topology, Heldermann Verlag Berlin, 1988. MR,1039321 (91c:54001)

[3] L. Gillman and M. Jerison, Rings of continuous functions, New York, 1960. MR0116199 $(22: 6994)$

[4] L. E. Pursell, comment: "Homomorphism on $C(\mathbb{R})$ ", Amer. Math. Monthly 94 (1987), no.7, 646. MR0935848 (89b:46065)

Department of Mathematics, Middle East Technical University, 06531 Ankara, TURKEY

E-mail address: zercan@metu.edu.tr

Department of Mathematics, Middle East Technical University, 06531 Ankara, TURKEY

E-mail address: osul@metu.edu.tr 